\title{
Physiological Changes in Glucose Differentially Modulate the Excitability of Hypothalamic Melanin-Concentrating Hormone and Orexin Neurons In Situ
}

\author{
Denis Burdakov, ${ }^{1}$ Oleg Gerasimenko, ${ }^{2}$ and Alexei Verkhratsky ${ }^{1}$ \\ ${ }^{1}$ Faculty of Life Sciences, University of Manchester, Manchester M13 9PT, United Kingdom, and 2Physiological Laboratory, University of Liverpool, \\ Liverpool L69 3BX, United Kingdom
}

\begin{abstract}
The physiological signaling mechanisms that link normal variations in body energy status to the activity of arousal- and metabolismregulating brain centers are not well understood. The melanin-concentrating hormone $(\mathrm{MCH})$ and orexin/hypocretin types of neurons of the lateral hypothalamus (LH) exert opposing effects on arousal and metabolism. We examined whether shifts in brain extracellular glucose that correspond to physiological changes in blood glucose can alter the electrical output of neurochemically and biophysically defined LH cells in mouse brain slices. Here, we show that physiologically relevant concentrations of glucose dose-dependently enhance the electrical excitability of $\mathrm{MCH}$ neurons by inducing depolarization and increasing membrane resistance. We also demonstrate that the same physiological shifts in glucose have the opposite effects on the electrical activity of orexin neurons. We propose that these direct actions of glucose on the arousal- and metabolism-regulating LH neurons play a key role in the translation of normal variations in body energy resources into appropriate changes in arousal and metabolism.
\end{abstract}

Key words: sleep; wakefulness; feeding; glucose; orexin; hypocretin; melanin-concentrating hormone

\section{Introduction}

In mammals, arousal is reduced after feeding and increased during fasting (Danguir and Nicolaidis, 1979; Dewasmes et al., 1989; Harnish et al., 1998; Yamanaka et al., 2003b), yet the physiological signaling mechanisms that couple body energy status to the activity of wakefulness- and metabolism-regulating brain centers are not well understood. The activity of neurons in the lateral hypothalamus (LH) is vital for normal sleep-wake behavior and body energy metabolism (Shimada et al., 1998; Saper et al., 2001; Willie et al., 2001; Sutcliffe and de Lecea, 2002; Taheri et al., 2002; Pissios and Maratos-Flier, 2003; Yamanaka et al., 2003b), and it has been recognized since the 1960s that some of these cells specifically respond to elevations in extracellular glucose with increased or decreased electrical activity (Oomura et al., 1969). However, the physiological significance of this potential arousalmodulating signaling pathway remained unsettled (Routh, 2002) because of the lack of knowledge about the functional identities of LH neurons whose electrical output is sensitive to physiologically relevant changes in ambient levels of glucose.

Two types of LH neurons, those expressing the neuropeptides melanin-concentrating hormone $(\mathrm{MCH})$ and orexins (hypocretins), recently emerged as critical regulators of sleep-wake behav-

Received Dec. 3, 2004; revised Jan. 18, 2005; accepted Jan. 24, 2005

This work was supported by the Royal Society and The Wellcome Trust. D.B. is a Royal Society Dorothy Hodgkin Research Fellow.

Correspondence should be addressed to Dr. Denis Burdakov, The University of Manchester, Faculty of Life Sciences, 1.124 Stopford Building, 0xford Road, Manchester M13 9PT, UK. E-mail: denis.burdakov@manchester.ac.uk. D01:10.1523/JNEUROSCI.4925-04.2005

Copyright $\odot 2005$ Society for Neuroscience $\quad$ 0270-6474/05/252429-05\$15.00/0 ior and energy balance (Shimada et al., 1998; Willie et al., 2001; Sutcliffe and de Lecea, 2002; Pissios and Maratos-Flier, 2003; Verret et al., 2003; Yamanaka et al., 2003b; Burdakov, 2004), but it has not been established whether the electrical activity of these vital cells is sensitive to physiological changes in glucose. Although the firing of isolated LH orexin neurons is inhibited by glucose in the 5-30 $\mathrm{mm}$ concentration range (Yamanaka et al., 2003 b), it is unknown whether this occurs in situ at physiologically relevant concentrations of brain glucose, which are $<5 \mathrm{~mm}$ (Silver and Erecinska, 1994; de Vries et al., 2003). The impact of changes in extracellular glucose on the electrical output of $\mathrm{MCH}$ neurons has not been explored.

In this study, we analyzed the electrical responses of $\mathrm{LH} \mathrm{MCH}$ and orexin neurons to physiological shifts in extracellular glucose in mouse brain slices, using patch-clamp recordings and postrecording immunocytochemistry.

\section{Materials and Methods}

Electrophysiology. Procedures involving animals were performed in accordance with the United Kingdom Animals (Scientific Procedures) Act of 1986. Brain slices containing the LH (bregma coordinates corresponding to between -1.2 and $-2 \mathrm{~mm}$ in adult mice) were prepared from male C57BL/6 mice (postnatal days 13-16), and whole-cell patch-clamp recordings and data analysis were performed as described previously (Burdakov et al., 2003). Experiments were performed at room temperature $\left(22-24^{\circ} \mathrm{C}\right)$. Extracellular solution was artificial CSF gassed with $95 \%$ $\mathrm{O}_{2}$ and $5 \% \mathrm{CO}_{2}$ and contained the following (in $\mathrm{mm}$ ): $118 \mathrm{NaCl}, 25$ $\mathrm{NaHCO}_{3}, 3 \mathrm{KCl}, 1.2 \mathrm{NaH}_{2} \mathrm{PO}_{4}, 2 \mathrm{CaCl}_{2}, 2 \mathrm{MgCl}_{2}$, and $0.2-5$ glucose. When the extracellular glucose concentration was altered, osmolarity was balanced with sucrose. Patch pipettes had tip resistances of 3-5 M $\Omega$ when filled with internal solution containing the following (in mM): 120 


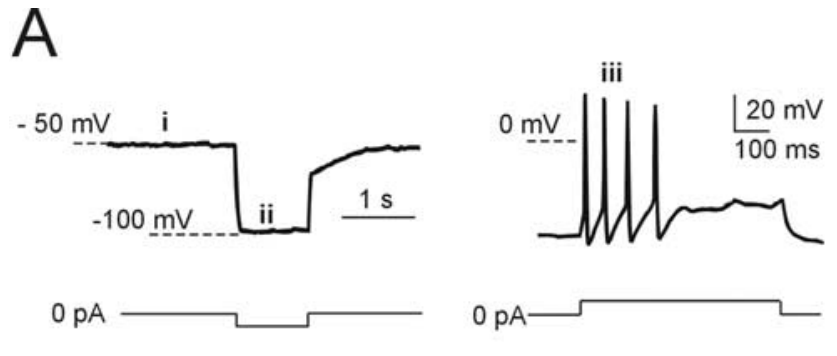

B

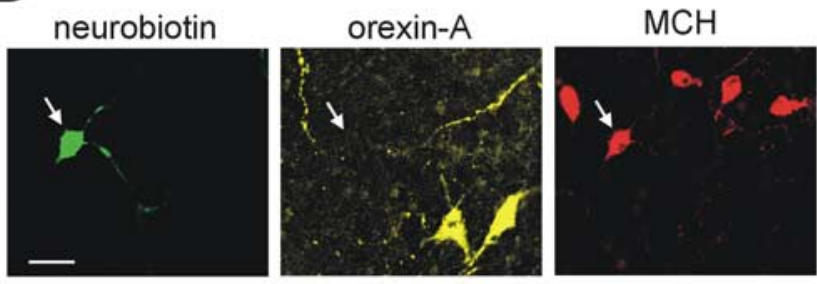

C

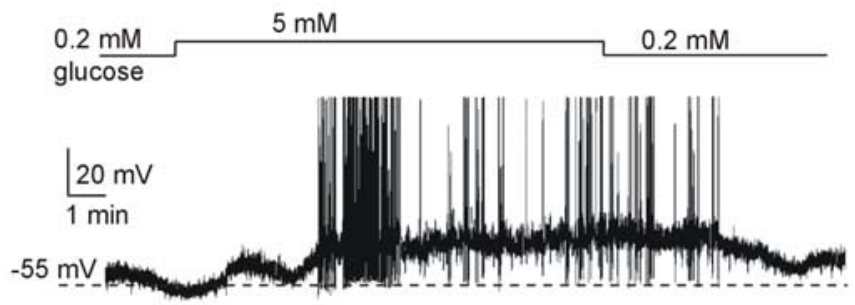

D

$0.2 \mathrm{mM}$ glucose $\quad 5 \mathrm{mM}$ glucose $\quad 0.2 \mathrm{mM}$ glucose
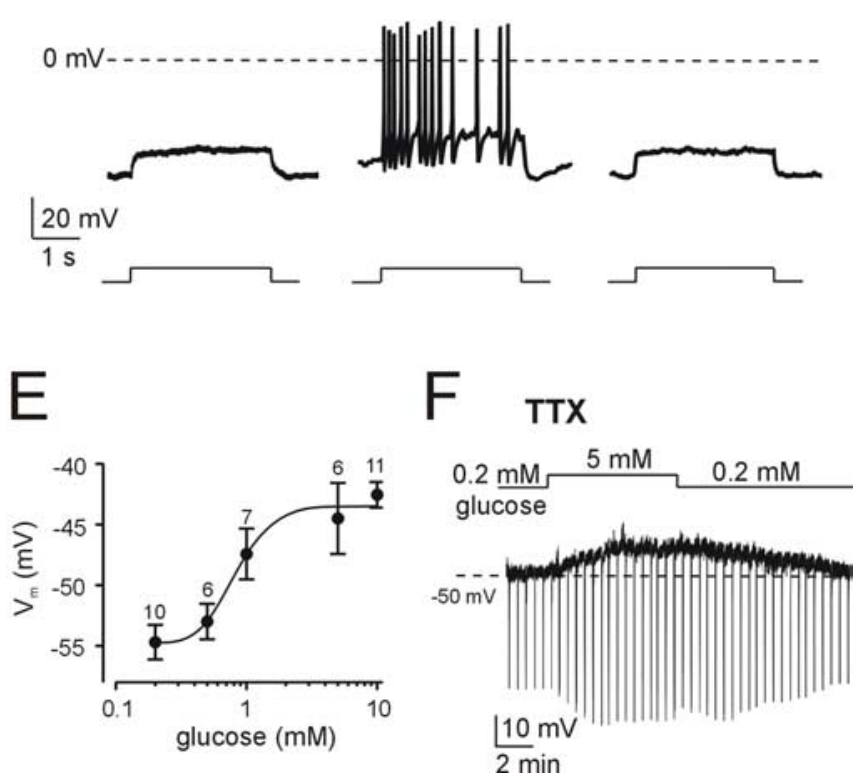

Figure 1. Electrical responses of $\mathrm{LH}$ MCH neurons to physiological changes in glucose. $\boldsymbol{A}$, Defining electrical signature of MCH neurons: absence of spontaneous firing (i), no H-currentmediated sag (ii), and spike-rate adaptation (iii). Current-clamp protocols used to elicit these responses are shown schematically below the corresponding traces. $\boldsymbol{B}$, Immunofluorescence imaging of the cell shown in $\boldsymbol{A}$, identified by Neurobiotin staining (green); the cell contains MCH (red) but not orexin-A (yellow). Scale bar, $20 \mu \mathrm{m}$. C, Glucose induced reversible depolarization and spiking in an MCH neuron. $\boldsymbol{D}$, Glucose enhanced spiking evoked by depolarizing current injection ( 20 pA for $3 \mathrm{~s}$; protocol shown schematically below the traces); this effect was revers-
K-gluconate, $10 \mathrm{KCl}, 0.1 \mathrm{EGTA}, 10 \mathrm{HEPES}, 4 \mathrm{~K}_{2}$-ATP, $1 \mathrm{Na}_{2} \mathrm{ATP}$, and 2 $\mathrm{MgCl}_{2}, \mathrm{pH} 7.3$ with KOH. All of the chemicals were from Sigma (Poole, Dorset, UK). Series resistance was $<10 \mathrm{M} \Omega$ throughout the recording and was not compensated. Data are given as mean \pm SEM. Concentration-response data were fitted with a modified Hill equation, $V_{\mathrm{m}}=$ $V_{\max }-\left(V_{\max }-V_{0}\right) /\left(1+\left([\text { glucose }] / \mathrm{EC}_{50}\right)^{h}\right)$, where $V_{\mathrm{m}}$ is the membrane potential, $V_{\max }$ and $V_{0}$ are saturation values of $V_{\mathrm{m}}$ as [glucose] is increased and decreased, respectively, $h$ is the Hill coefficient, and $\mathrm{EC}_{50}$ (or $\mathrm{IC}_{50}$ ) is the concentration of glucose at which the $V_{\mathrm{m}}$ change is halfmaximal. Parameter values used to obtain specific fits of the equation to the data are given in the relevant figure legends (see Figs. $1 E, 2 E$ ).

Immunocytochemistry and confocal microscopy. For postrecording neurochemical identification of LH neurons, $0.2 \%$ Neurobiotin tracer (Vector Laboratories, Peterborough, UK) was added to the pipette solution. Neurons were maintained in the whole-cell configuration for 5-10 min to allow thorough infusion of Neurobiotin, after which the patch pipette was gently withdrawn via the outside-out configuration. Slices were then fixed in 4\% paraformaldehyde (Sigma) in PBS, pH 7.4, for $1 \mathrm{~h}$ at room temperature, incubated with primary antibodies overnight, and then incubated with fluorescent secondary antibodies for $3 \mathrm{~h}$, following a previously published protocol (Wolfart et al., 2001). The primary antibodies and labels were Neurobiotin, rabbit anti-orexin-A (1:250) (Phoenix Pharmaceuticals, Karlsruhe, Germany), and chicken anti-MCH (1:250) (BMA Biomedicals, Augst, Switzerland). The corresponding secondary antibodies and labels were streptavidin-cyanine 2 (Cy2) (1:500) (Amersham Biosciences, Bucks, UK), goat anti-rabbit-Cy5 (1:500) (Amersham Biosciences), and goat anti-chicken-Alexa Fluor 555 (1:500) (Molecular Probes, Eugene, OR). The specificity of the antibodies we used was established in previous studies (Chen et al., 1999; Pereira-da-Silva et al., 2003). Slices were analyzed using a Leica Microsystems (Mannheim, Germany) TCS SP2 confocal laser-scanning microscope. Cy2 (shown in green; see Figs. $1 B, 2 B$ ) was excited with an argon laser at $488 \mathrm{~nm}$, and fluorescence emission was collected at 505-530 nm. Alexa Fluor 555 (red) and Cy5 (yellow) were excited with HeNe lasers at 543 and $633 \mathrm{~nm}$, respectively, and emission was detected at $560-615$ and $650-700 \mathrm{~nm}$. To achieve optimal separation of fluorescent signals, the sequential "between lines" scanning mode of the microscope was used.

\section{Results}

Identification of $\mathrm{MCH}$ and orexin neurons in brain slices

We identified $\mathrm{MCH}$ and orexin cells by their distinctive electrophysiological properties and postrecording immunocytochemistry. $\mathrm{MCH}$ neurons are electrically silent, do not display H-current-mediated depolarization, and exhibit pronounced spike-rate adaptation (Eggermann et al., 2003; van den Pol et al., 2004). Using postrecording triple-label immunocytochemistry to correlate electrophysiological signature with expression of $\mathrm{MCH}$ and orexin peptides (see Materials and Methods), we confirmed that all LH neurons that displayed this combination of properties (Fig. $1 \mathrm{~A})$ contained $\mathrm{MCH}$ and not orexin $(n=30$ of 30) (Fig. $1 B$ ).

In contrast to $\mathrm{MCH}$ neurons, orexin neurons exhibit tonic spontaneous firing, $\mathrm{H}$-currents, low-threshold spike on recovery from hyperpolarization, and little spike-rate adaptation (Li et al., 2002; Eggermann et al., 2003; Yamanaka et al., 2003a; Burdakov et al., 2004). We confirmed by postrecording immunocytochemistry that all LH neurons displaying the latter set of properties (Fig. 2A) contained orexin and not $\mathrm{MCH}$ peptides ( $n=35$ of 35)

ible after glucose washout. $E$, Dose-response relationship of glucose-induced depolarization of $M C H$ cells $\left(E C_{50}=0.8 \mathrm{~mm} ; h=3.2 ; V_{\max }=-43.5 \mathrm{mV} ; V_{0}=-54.8 \mathrm{mV}\right.$; the general equation of the fit is given in Materials and Methods). Numbers of cells are indicated above corresponding points. $\boldsymbol{F}$, In the presence of tetrodotoxin (TTX) (300 nM), glucose induced depolarization and increased membrane resistance (the latter effect is manifested as increased amplitude of membrane potential responses to hyperpolarizing current pulses; $40 \mathrm{pA}$ for $500 \mathrm{~ms}$ at 30 s intervals). 

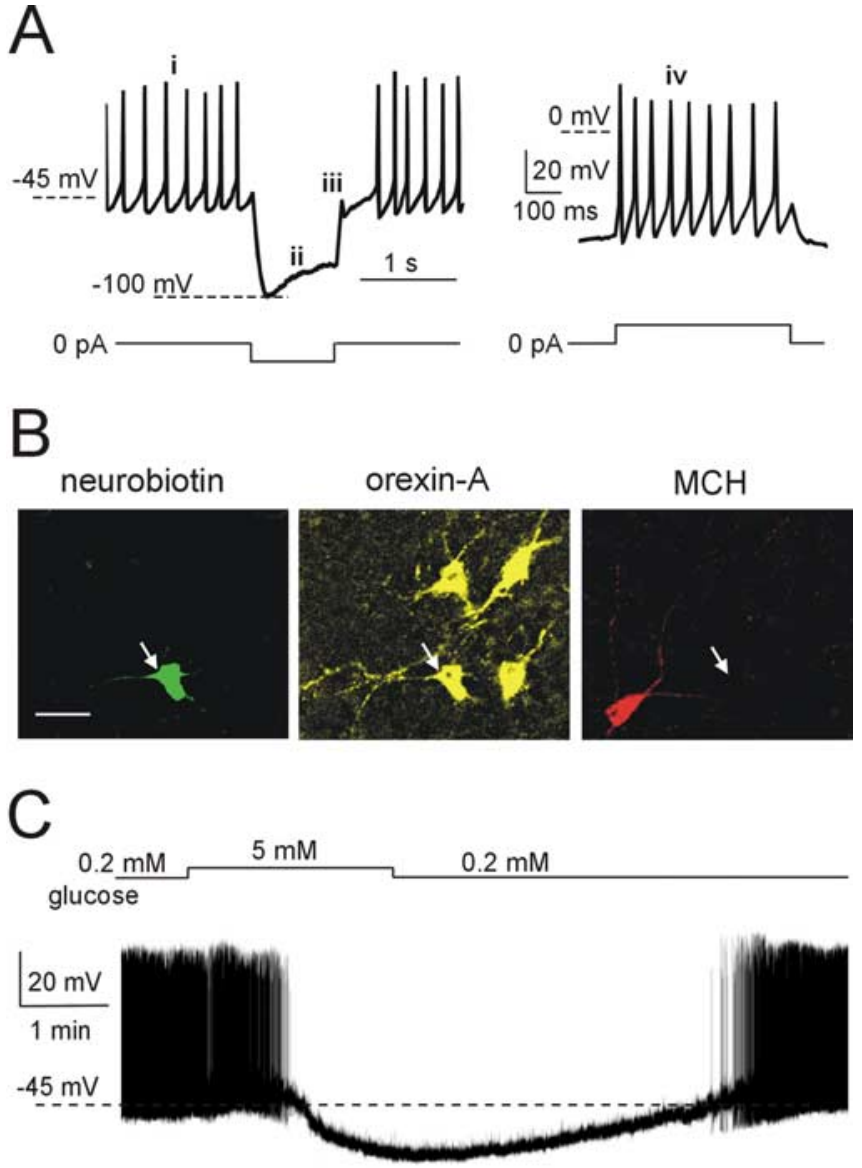

$\mathrm{D}$
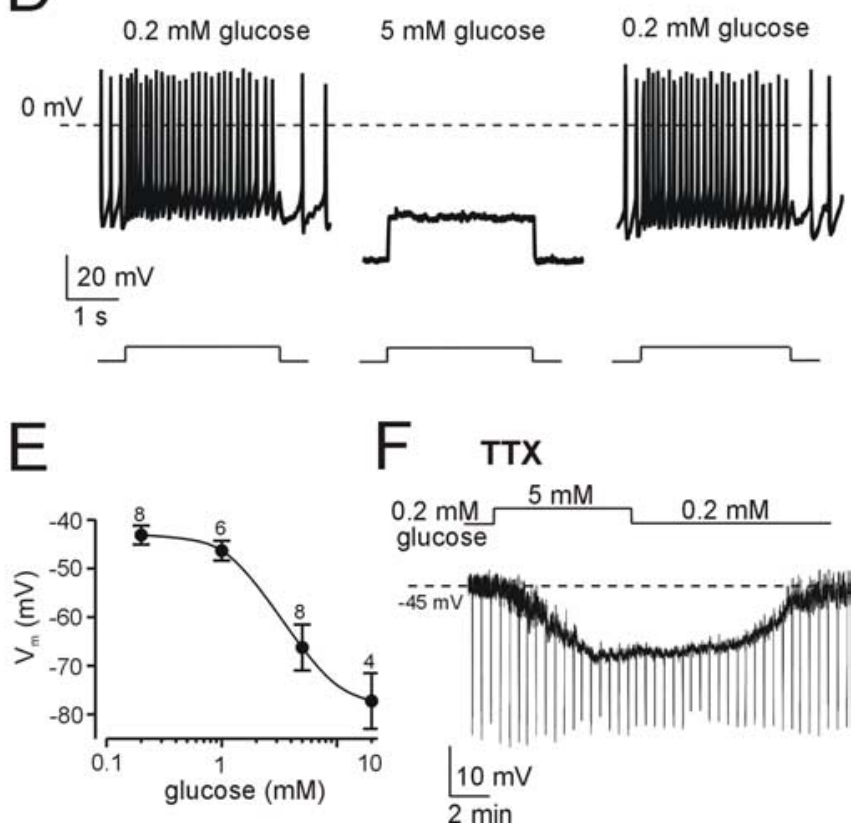

Figure 2. Electrical responses of $\mathrm{LH}$ orexin neurons to physiological changes in glucose. $\boldsymbol{A}$, Defining electrical signature of orexin neurons: tonic spontaneous firing (i), H-currentmediated sag (ii), low-threshold spike (iii), and little spike-rate adaptation (iv). Current-clamp protocols used to elicit these responses are shown schematically below corresponding traces. $\boldsymbol{B}$, Immunofluorescence imaging of the cell shown in $\boldsymbol{A}$, identified by Neurobiotin staining (green); the cell contains orexin-A (yellow) but not MCH (red). Scale bar, $20 \mu \mathrm{m}$. C, Glucose induced reversible hyperpolarization and inhibited spiking in an orexin neuron. $\boldsymbol{D}$, Glucose suppressed the spiking response to depolarizing current injection ( 40 pA for $3 \mathrm{~s}$; protocol shown schemat-
(Fig. $2 B$ ). Our qualitative electrophysiological criteria (Figs. $1 A$, $2 A$ ) therefore provide a highly reliable way of identifying $\mathrm{MCH}$ and orexin neurons in our in situ preparation.

Physiological concentrations of glucose excite $\mathrm{MCH}$ neurons In vivo, extracellular glucose concentration in the brain varies between $\sim 0.2 \mathrm{~mm}$ during systemic hypoglycemia and $\sim 5 \mathrm{~mm}$ during hyperglycemia [measurements from rat brain (Silver and Erecinska, 1994)]. Changing extracellular glucose from 0.2 to 5 mM reversibly depolarized MCH neurons ( $n=15$ of 18) (Fig. $1 C$ ). This depolarization was accompanied by the appearance of spiking responses to depolarizing current pulses that failed to induce action potentials in $0.2 \mathrm{~mm}$ glucose $(n=15$ of 15$)$ (Fig. $1 D)$ and by induction of spontaneous firing in 4 of $15 \mathrm{MCH}$ neurons (Fig. 1C). Glucose-induced depolarization of $\mathrm{MCH}$ neurons was dose dependent with half-maximal effect at $0.8 \mathrm{~mm}$ (Fig. $1 E$ ) and was associated with an increase in membrane resistance (calculated from membrane potential changes in response to a series of 10-40 pA hyperpolarizing current pulses, such as those shown in Fig. $1 F$ ). Membrane resistance increased by $74 \pm 18 \%$ in $5 \mathrm{~mm}$ glucose compared with control values in $0.2 \mathrm{~mm}$ glucose measured in the same cell $(n=8)$. The largest resistance increase observed was from 400 to $1100 \mathrm{M} \Omega$, and the smallest was from 650 to $800 \mathrm{M} \Omega$ in 0.2 and $5 \mathrm{~mm}$ glucose, respectively. Reversible glucose-induced depolarization and resistance increase persisted when neurons were synaptically isolated with tetrodotoxin $(n=$ 4) (Fig. $1 F)$, consistent with a direct postsynaptic action of glucose on $\mathrm{MCH}$ neurons.

\section{Physiological concentrations of glucose inhibit} orexin neurons

The same physiological changes in glucose had the opposite effects on LH orexin neurons. Elevating glucose from 0.2 to $5 \mathrm{mM}$ induced hyperpolarization and suppressed both spontaneous (Fig. 2C) and evoked (Fig. 2D) firing in 20 of 21 orexin neurons. Glucose-induced hyperpolarization of orexin neurons was concentration dependent with half-maximal response at $3.5 \mathrm{~mm}$ (Fig. $2 E$ ) and was accompanied by a pronounced decrease in membrane resistance (Fig. 2 F). Membrane resistance fell by $66 \pm 3 \%$ in $5 \mathrm{~mm}$ glucose compared with control values in $0.2 \mathrm{~mm}$ glucose measured in the same cell $(n=7)$. The largest resistance decrease was from 1000 to $250 \mathrm{M} \Omega$, and the smallest was from 1160 to 530 $\mathrm{M} \Omega$ in 0.2 and $5 \mathrm{~mm}$ glucose, respectively. The hyperpolarization and resistance decrease persisted in tetrodotoxin $(n=5)$ (Fig. $2 F$ ), indicating a direct postsynaptic action of glucose on orexin neurons.

\section{Discussion}

Effects of glucose on $\mathrm{MCH}$ and orexin neurons

In this study, we show for the first time that physiologically relevant concentrations of glucose directly enhance the electrical excitability of $\mathrm{MCH}$ neurons by causing depolarization and increasing membrane resistance but inhibit the firing of orexin neurons by triggering hyperpolarization and decreasing membrane resistance. Crucially, our concentration-response analysis (Figs. 1E,

ically below the traces); this effect was reversible after glucose washout. $\boldsymbol{E}$, Dose-response relationship of glucose-induced hyperpolarization of orexin cells $\left(\mathrm{IC}_{50}=3.5 \mathrm{~mm} ; h=1.8\right.$; $V_{\max }=-78.8 \mathrm{mV} ; V_{0}=-42.9 \mathrm{mV}$; the general equation of the fit is given in Materials and Methods). Numbers of cells are indicated above corresponding points. $\boldsymbol{F}$, In the presence of tetrodotoxin (TTX) (300 nM), glucose induced hyperpolarization and decreased membrane resistance (resistance was monitored as described in Fig. 1F). 
$2 E)$ showed that these effects were steepest within the physiological window of brain glucose levels (Silver and Erecinska, 1994) and would be expected to occur in response to variations in hypothalamic glucose associated with changes in food intake (de Vries et al., 2003).

The effects of glucose on membrane resistance imply that glucose suppresses an inhibitory ionic conductance in $\mathrm{MCH}$ neurons but activates an inhibitory conductance in orexin neurons. In other neurons that electrically respond to changes in glucose, glucose-induced excitation is thought to be attributable to the closure of ATP-sensitive $\mathrm{K}^{+}$channels, whereas glucose-induced inhibition has been proposed to involve activation of $\mathrm{a} \mathrm{Cl}^{-}$conductance (Levin et al., 2004; Song and Routh, 2005). The biophysical identities of glucose-modulated conductances in $\mathrm{MCH}$ and orexin neurons remain to be determined. Another important subject for future investigation is the developmental profile of the glucose responses; it would be of particular interest to compare glucose sensitivity of LH neurons from young mice used here with that of LH cells from older animals.

Our recordings show that stimulating orexin neurons with 10 mM glucose for several minutes caused them to assume hyperpolarized membrane potentials (approximately $-74 \mathrm{mV}$ on average) and completely abolished their firing (Fig. 2E). In contrast, other recent reports indicate that after LH brain slices have been maintained in $10 \mathrm{~mm}$ glucose for a long time (throughout postslicing recovery and subsequent experiments), orexin neurons exhibit depolarized membrane potentials and spontaneous firing (Li et al., 2002; Eggermann et al., 2003; Yamanaka et al., 2003a; Burdakov et al., 2004). One potential explanation for this apparent difference between short- and long-term responses to changes in glucose levels is that orexin neurons could possess adaptive homeostatic mechanisms (Marder and Prinz, 2002), which, when faced with sustained changes in glucose, may gradually return the electrical properties of orexin cells to an intrinsic set point, such as the depolarized and spontaneously firing state (Eggermann et al., 2003).

\section{Implications for wakefulness and energy balance}

Both MCH and orexin neurons of the LH innervate multiple brain areas that control appetite, metabolism, and sleep-wake behavior (Bittencourt et al., 1992; Peyron et al., 1998). MCH promotes sleep (Verret et al., 2003) and suppresses metabolism (Segal-Lieberman et al., 2003), and its knock-out produces a lean mouse (Shimada et al., 1998). This implies that the excitatory actions of glucose on $\mathrm{MCH}$ neurons (Fig. 1) may promote rest and energy conservation when body energy resources are high (e.g., after feeding). Conversely, decreased excitability of $\mathrm{MCH}$ neurons caused by a fall in glucose would be expected to suppress sleepiness and promote activity, which is advantageous for effective food seeking.

In contrast to $\mathrm{MCH}$, orexins promote wakefulness and stimulate metabolism, whereas destruction of orexin neurons causes narcolepsy (Willie et al., 2001; Sutcliffe and de Lecea, 2002; Taheri et al., 2002). It was proposed recently that disinhibition of orexin neurons caused by falling levels of glucose may mediate the increased wakefulness and activity that accompany fasting (Yamanaka et al., 2003b), yet the physiological significance of this pathway remained controversial because inhibition of the electrical output of orexin neurons by physiological concentrations of glucose in situ has not been demonstrated. Our new results (Fig. 2) now provide the crucial physiological evidence that orexin neurons in situ indeed dramatically alter their spontaneous and evoked electrical output in response to changes in ambient glu- cose that correspond to different metabolic states in vivo (Silver and Erecinska, 1994; de Vries et al., 2003).

In summary, our data offer an integrated biophysical and neurochemical explanation for how LH glucose-sensing neurons translate subtle physiological variations in glucose levels into appropriate adaptive changes in arousal and energy metabolism. This may also be relevant to understanding the pathogenesis of narcolepsy and body-weight disorders.

\section{References}

Bittencourt JC, Presse F, Arias C, Peto C, Vaughan J, Nahon JL, Vale W, Sawchenko PE (1992) The melanin-concentrating hormone system of the rat brain: an immuno- and hybridization histochemical characterization. J Comp Neurol 319:218-245.

Burdakov D (2004) Electrical signaling in central orexin/hypocretin circuits: tuning arousal and appetite to fit the environment. The Neuroscientist 10:286-291.

Burdakov D, Liss B, Ashcroft FM (2003) Orexin excites GABAergic neurons of the arcuate nucleus by activating the sodium-calcium exchanger. J Neurosci 23:4951-4957.

Burdakov D, Alexopoulos H, Vincent A, Ashcroft FM (2004) Low-voltageactivated A-current controls the firing dynamics of mouse hypothalamic orexin neurons. Eur J Neurosci 20:3281-3285.

Chen CT, Dun SL, Kwok EH, Dun NJ, Chang JK (1999) Orexin A-like immunoreactivity in the rat brain. Neurosci Lett 260:161-164.

Danguir J, Nicolaidis S (1979) Dependence of sleep on nutrients' availability. Physiol Behav 22:735-740.

de Vries MG, Arseneau LM, Lawson ME, Beverly JL (2003) Extracellular glucose in rat ventromedial hypothalamus during acute and recurrent hypoglycemia. Diabetes 52:2767-2773.

Dewasmes G, Duchamp C, Minaire Y (1989) Sleep changes in fasting rats. Physiol Behav 46:179-184.

Eggermann E, Bayer L, Serafin M, Saint-Mleux B, Bernheim L, Machard D, Jones BE, Muhlethaler M (2003) The wake-promoting hypocretinorexin neurons are in an intrinsic state of membrane depolarization. J Neurosci 23:1557-1562.

Harnish MJ, Greenleaf SR, Orr WC (1998) A comparison of feeding to cephalic stimulation on postprandial sleepiness. Physiol Behav 64:93-96.

Levin BE, Routh VH, Kang L, Sanders NM, Dunn-Meynell AA (2004) Neuronal glucosensing: what do we know after 50 years? Diabetes 53:2521-2528.

Li Y, Gao XB, Sakurai T, van den Pol AN (2002) Hypocretin/orexin excites hypocretin neurons via a local glutamate neuron-A potential mechanism for orchestrating the hypothalamic arousal system. Neuron 36:1169-1181.

Marder E, Prinz AA (2002) Modeling stability in neuron and network function: the role of activity in homeostasis. BioEssays 24:1145-1154.

Oomura Y, Ono T, Ooyama H, Wayner MJ (1969) Glucose and osmosensitive neurones of the rat hypothalamus. Nature 222:282-284.

Pereira-da-Silva M, Torsoni MA, Nourani HV, Augusto VD, Souza CT, Gasparetti AL, Carvalheira JB, Ventrucci G, Marcondes MC, Cruz-Neto AP, Saad MJ, Boschero AC, Carneiro EM, Velloso LA (2003) Hypothalamic melanin-concentrating hormone is induced by cold exposure and participates in the control of energy expenditure in rats. Endocrinology 144:4831-4840.

Peyron C, Tighe DK, van den Pol AN, de Lecea L, Heller HC, Sutcliffe JG, Kilduff TS (1998) Neurons containing hypocretin (orexin) project to multiple neuronal systems. J Neurosci 18:9996-10015.

Pissios P, Maratos-Flier E (2003) Melanin-concentrating hormone: from fish skin to skinny mammals. Trends Endocrinol Metab 14:243-248.

Routh VH (2002) Glucose-sensing neurons: are they physiologically relevant? Physiol Behav 76:403-413.

Saper CB, Chou TC, Scammell TE (2001) The sleep switch: hypothalamic control of sleep and wakefulness. Trends Neurosci 24:726-731.

Segal-Lieberman G, Bradley RL, Kokkotou E, Carlson M, Trombly DJ, Wang X, Bates S, Myers Jr MG, Flier JS, Maratos-Flier E (2003) Melaninconcentrating hormone is a critical mediator of the leptin-deficient phenotype. Proc Natl Acad Sci USA 100:10085-10090.

Shimada M, Tritos NA, Lowell BB, Flier JS, Maratos-Flier E (1998) Mice lacking melanin-concentrating hormone are hypophagic and lean. Nature 396:670-674. 
Silver IA, Erecinska M (1994) Extracellular glucose concentration in mammalian brain: continuous monitoring of changes during increased neuronal activity and upon limitation in oxygen supply in normo-, hypo-, and hyperglycemic animals. J Neurosci 14:5068-5076.

Song Z, Routh VH (2005) Differential effects of glucose and lactate on glucosensing neurons in the ventromedial hypothalamic nucleus. Diabetes 54:15-22.

Sutcliffe JG, de Lecea L (2002) The hypocretins: setting the arousal threshold. Nat Rev Neurosci 3:339-349.

Taheri S, Zeitzer JM, Mignot E (2002) The role of hypocretins (orexins) in sleep regulation and narcolepsy. Annu Rev Neurosci 25:283-313.

van den Pol AN, Acuna-Goycolea C, Clark KR, Ghosh PK (2004) Physiological properties of hypothalamic $\mathrm{MCH}$ neurons identified with selective expression of reporter gene after recombinant virus infection. Neuron 42:635-652.

Verret L, Goutagny R, Fort P, Cagnon L, Salvert D, Leger L, Boissard R, Salin P, Peyron C, Luppi PH (2003) A role of melanin-concentrating hor- mone producing neurons in the central regulation of paradoxical sleep. BMC Neurosci 4:19.

Willie JT, Chemelli RM, Sinton CM, Yanagisawa M (2001) To eat or to sleep? Orexin in the regulation of feeding and wakefulness. Annu Rev Neurosci 24:429-458.

Wolfart J, Neuhoff H, Franz O, Roeper J (2001) Differential expression of the small-conductance, calcium-activated potassium channel SK3 is critical for pacemaker control in dopaminergic midbrain neurons. J Neurosci 21:3443-3456.

Yamanaka A, Muraki Y, Tsujino N, Goto K, Sakurai T (2003a) Regulation of orexin neurons by the monoaminergic and cholinergic systems. Biochem Biophys Res Commun 303:120-129.

Yamanaka A, Beuckmann CT, Willie JT, Hara J, Tsujino N, Mieda M, Tominaga M, Yagami K, Sugiyama F, Goto K, Yanagisawa M, Sakurai T (2003b) Hypothalamic orexin neurons regulate arousal according to energy balance in mice. Neuron 38:701-713. 